\title{
Primary Ocular and Central Nervous System Malignant Lymphoma First Manifested as Uveitis: Possible Role of Single Photon Emission Computed Tomography with $\mathrm{N}$-isopropyl- ${ }^{123}$ I-p-iodoamphetamine in the Diagnostic Procedure
}

\author{
Toshihiro Yoshizawa, Yasushi Makiyama, Keiko Nakazato*, Hiroshi KoJima**, \\ Sachiko Honmura*, Hidehiro Mizusawa and Shin'ichi SHOJI
}

\begin{abstract}
A case of primary ocular and central nervous system malignant lymphoma was reported which was first manifested as bilateral uveitis ten months before the neurological symptoms appeared. The lesion of lymphoma in the central nervous system was clearly demonstrated as a heavy accumulation of $\mathrm{N}$-isopropyl- ${ }^{123} \mathrm{I}$-p-iodoamphetamine by single photon emission computed tomography. Since most brain tumors reportedly show a low uptake of $\mathrm{N}$-isopropyl- ${ }^{123} \mathrm{I}-\mathrm{p}$ iodoamphetamine, single photon emission computed tomography with $\mathrm{N}$-isopropyl- ${ }^{123} \mathrm{I}-\mathrm{p}$ iodoamphetamine could be helpful in making the diagnosis, as well as in visualizing primary central nervous system malignant lymphoma.
\end{abstract}

(Internal Medicine 33: 92-96, 1994)

Key words: magnetic resonance imaging (MRI), computed tomography (CT), cytology, $\beta_{2^{-}}$ microglobulin, cerebrospinal fluid

\section{Introduction}

Uveitis is known to precede the symptoms of primary central nervous system (CNS) malignant lymphoma. Since most of these ocular lesions have been reported to represent infiltrations of malignant lymphoma, these cases have been termed "masquerade syndrome" and classified as primary ocular and CNS malignant lymphoma which are assumed to have multifocal neoplastic origin (1-8). In such cases, it is difficult to make a diagnosis of malignant lymphoma until the CNS symptoms appear.

Here we describe a case of primary ocular and central nervous system malignant lymphoma in which bilateral uveitis appeared ten months before the CNS symptoms emerged. In this case, single photon emission computed tomography (SPECT) with N-isopropyl- ${ }^{123}$ I-p-iodoamphetamine $\left({ }^{123}\right.$ I-IMP) clearly demonstrated a dense accumulation of ${ }^{123}$ I-IMP in the CNS lesion of malignant lymphoma. Most brain tumors are reported to show a low uptake of ${ }^{123}$ I-IMP (9), so SPECT with

${ }^{123}$ I-IMP could be helpful in making the diagnosis of primary CNS malignant lymphoma.

\section{Case Report}

A 37-year-old woman noticed blurred vision in August 1990 and was diagnosed as having idiopathic bilateral uveitis. As her visual acuity was gradually worsening, oral corticosteroid therapy was started in March 1991. Her visual symptoms rapidly improved. In June 1991, when steroid therapy was tapered off, she developed headaches, unsteadiness of gait and blurred vision, and was therefore admitted to our hospital. She was afebrile. Ophthalmological examination on admission disclosed bilateral decrease in visual acuity due to posterior uveitis. Neurological examination showed horizontal gaze nystagmus, slight dysmetria in her left arm and gait ataxia. There were no disturbances of higher function nor meningeal signs observed. Examination of cerebrospinal fluid (CSF) indicated slight pleocytosis (10 mononuclear cells and 6 atypical cells per $\mu \mathrm{l})$, a normal protein level $(41 \mathrm{mg} / \mathrm{dl})$ and an increased level of $\beta_{2}$-microglobulin $(4,571 \mu \mathrm{g} / \mathrm{l})$. Magnetic resonance imaging (MRI) performed immediately after admission revealed several intracranial lesions. One of them was located in the splenium, which showed low intensity on a T1-weighted image (Fig. 1A) and irregular intensity surrounded by high

From the Department of Neurology, *Ophthalmology and **Hematology, Institute of Clinical Medicine, University of Tsukuba, Tsukuba Received for publication June 15, 1993; Accepted for publication December 2, 1993

Reprint requests should be addressed to Dr. Toshihiro Yoshizawa, the Department of Neurology, Institute of Clinical Medicine, University of Tsukuba, 1-1-1 Tennodai, Tsukuba 305 

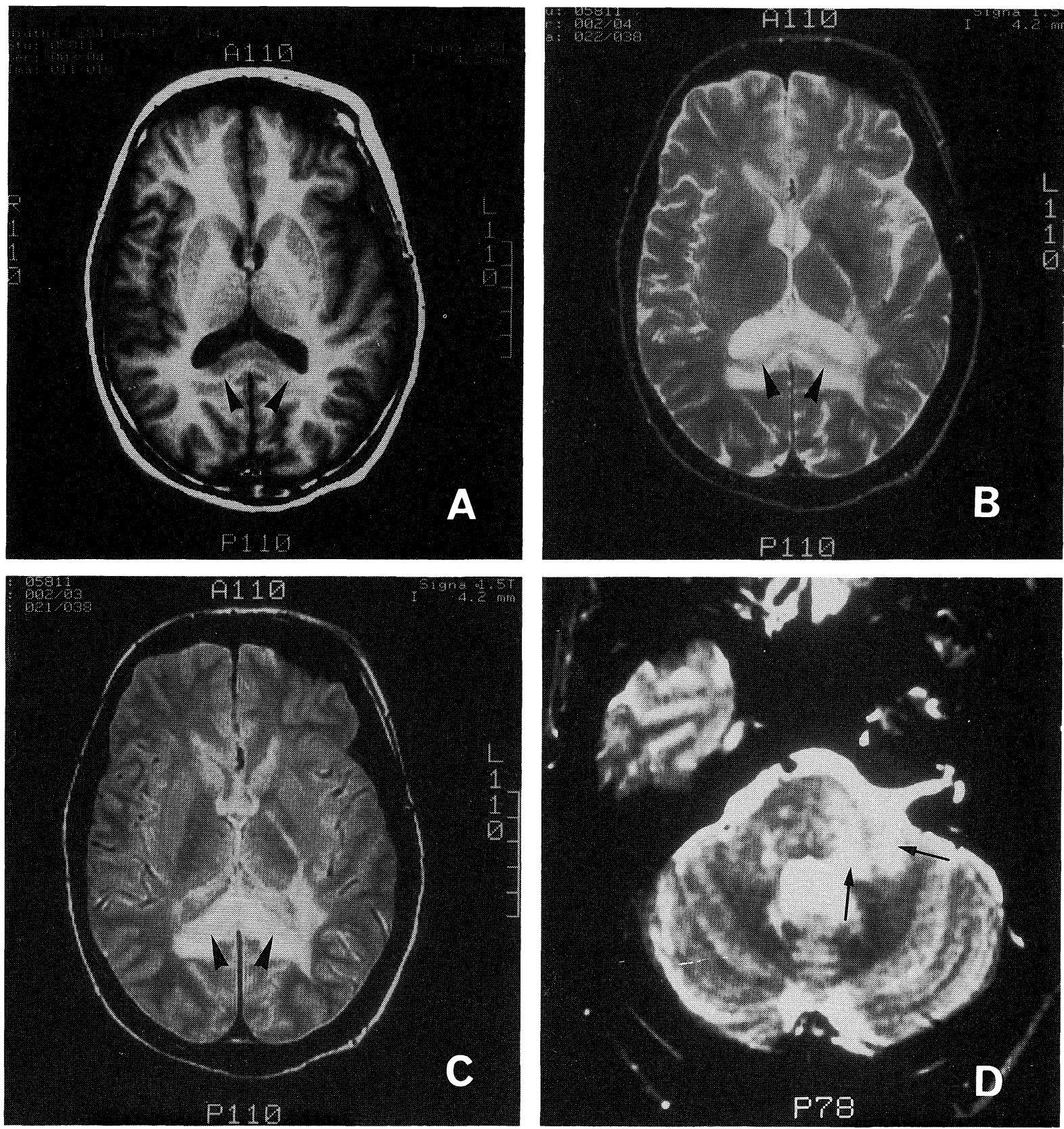

Fig. 1. Demonstration of intracranial lesions of primary central nervous system malignant lymphoma in the MR scans performed immediately after admission. A) T1-weighted (TR $400 \mathrm{~ms}$, TE $14 \mathrm{~ms}$ ), B) T2-weighted (TR $3000 \mathrm{~ms}$, TE $90 \mathrm{~ms}$ ) and C) proton (TR $3000 \mathrm{~ms}$, TE $30 \mathrm{~ms}$ ) images disclose the lesion at the splenium (arrow heads). D) Multiple high intense lesions are mainly located in the left cerebellar peduncle on a T2-weighted image (TR $3000 \mathrm{~ms}$, TE $90 \mathrm{~ms}$ ). The arrows indicate the lesions.

intensity on a T2-weighted image (Fig. 1B) and proton image (Fig. 1C). The splenium lesion was markedly enhanced by gadolinium (Gd)-DTPA and was clearly differentiated from the surrounding brain tissue (Fig. 2A). In addition, a T2-weighted image showed multiple high intense lesions located mainly in the left middle cerebellar peduncle (Fig. 1D). The splenium lesion was also enhanced on the contrast-enhanced computed tomographic scan (CT scan) (data not shown). Furthermore, we performed ${ }^{123}$ I-IMP SPECT at 15 minutes and 3 hours after intravenous administration of ${ }^{123}$ I-IMP (222 MBq) with HEADTOME SET-050 (Shimadzu Co., Kyoto). The first image obtained 15 minutes after administration showed unusually heavy accumulation of IMP in the splenium (Fig. 2B), and this became more clear 3 hours after administration of IMP (Fig. $2 \mathrm{C}$ ). Since these radiological findings strongly suggested that the CNS lesions were malignant lymphoma, we collected CSF cells by centrifugation and stained these cells with Wright's stain. Microscopically, many large lymphoid cells with large 

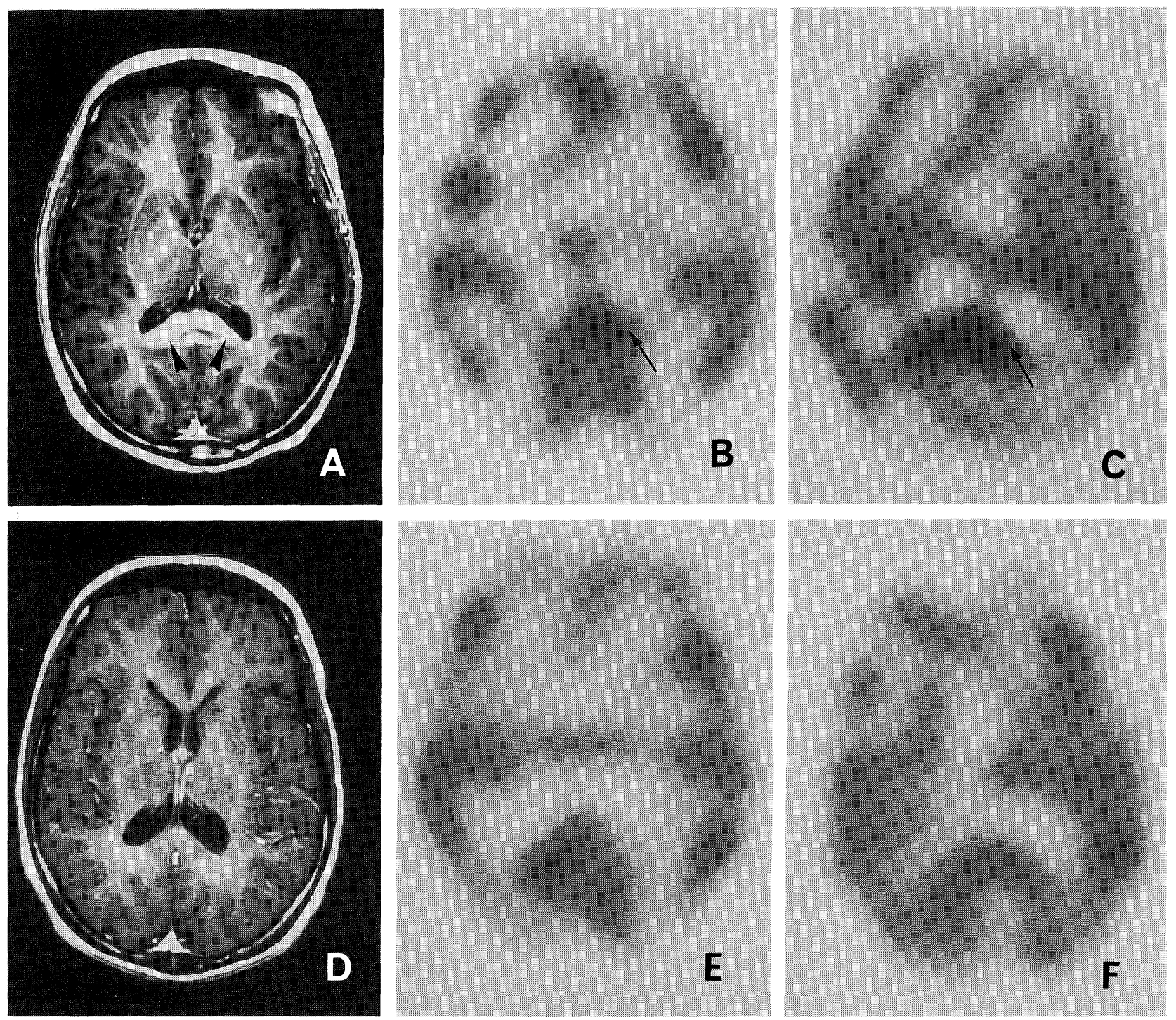

Fig. 2. ${ }^{123}$ I-IMP SPECT images compared with Gd-DTPA enhanced MRI before (A-C) and after treatment (D-F). A) Gd-DTPA-enhanced T1weighted image (TR $400 \mathrm{~ms}$, TE $14 \mathrm{~ms}$ ) shows dense enhancement at the splenium lesion (arrowhead). B) Early and C) delayed images of ${ }^{123}$ I-IMP SPECT. Dense accumulation of IMP is observed at the splenium lesion (arrows). After treatment, enhancement by Gd-DTPA in the MRI disappeared. Moreover, accumulation of IMP in the splenium decreased in the early and delayed images.

hyperchromatic nuclei consistent with large-cell lymphoma were found (Fig. 3). Other laboratory findings were normal. Gallium scintigraphy showed increased uptake in both eyes and splenium. However, bone-marrow biopsy, abdominal ultrasonography, thoracic and abdominal CT scans did not reveal any systemic lesions. From these findings, we diagnosed this case as primary ocular and central nervous system malignant lymphoma first manifested as uveitis.

She was initially treated with radiotherapy (RT) to both eyes (30 Gy) and intrathecal administration of corticosteroid and methotrexate (MTX). A combination regimen using pre-RT systemic MTX and intra-Ommaya MTX, whole-brain RT and post-RT high-dose cytosine arabinoside (Ara-C) was planned to follow (10). After she received systemic MTX (total $3 \mathrm{~g}$ ) and intrathecal MTX (total $96 \mathrm{mg}$ ), we performed MRI and ${ }^{123} \mathrm{I}$ IMP SPECT again. On a Gd-DTPA enhanced T1-weighted image, the splenium lesion was no longer enhanced by GdDTPA (Fig. 2D). In addition, accumulation of IMP in the splenium markedly decreased both in the early image (Fig. 2E) and delayed image (Fig. 2F).

Since she developed pneumonitis after MTX treatment, we exchanged the former protocol for a combination regimen using chemotherapy (dexamethasone, cisplatin and Ara-C) and wholebrain RT. The treatment was successful. She was discharged in November 1991. 


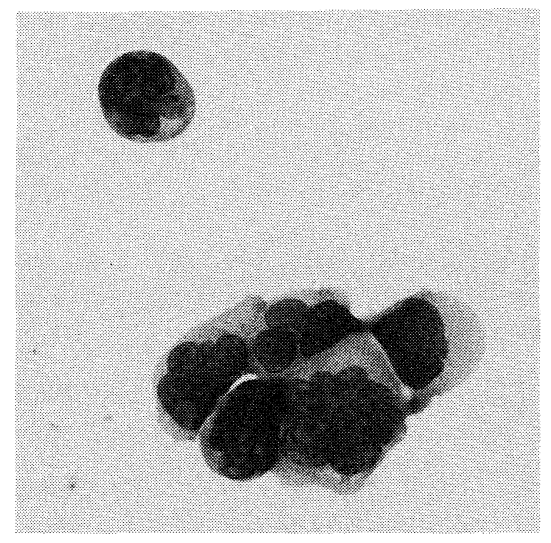

Fig. 3. Atypical cells in CSF collected by centrifugation and stained with Wright's stain $(\times 400)$. Many large lymphoid cells with large hyperchromatic nuclei, which were consistent with large-cell lymphoma, were found.

\section{Discussion}

Several reports describe that primary CNS malignant lymphoma could involve ocular tissues and simulate uveitis (18). Because ocular symptoms are reported to often precede CNS symptoms in such cases, diagnosis of malignant lymphoma seemed difficult before CNS symptoms appeared. Although cytologic examination of vitreous aspirate was reported to be useful for the diagnosis of malignant lymphoma in the opthalmological field $(3,9)$, it is good to always consider that uveitis can be an early manifestation of primary ocular and CNS malignant lymphoma, and to carefully follow-up the neurological examination of these patients.

For the diagnosis of primary CNS malignant lymphoma, an increased $\beta_{2}$-microglobulin level in CSF (11) and neuroradiological findings on CT $(12,13)$ or MRI (14) have been reported to be useful. Actually, the increased level of $B_{2}$ microglobulin in CSF and marked enhancement by contrast enhanced material on CT scans and by Gd-DTPA on MR scans were observed in the present case. In addition to these findings, a heavy accumulation of ${ }^{123}$ I-IMP was recognized both on the early and delayed images by SPECT.

Since intravenously administered ${ }^{123}$ I-IMP passes through the blood-brain barrier and remains in the brain tissue for several hours, the distribution of ${ }^{123}$ I-IMP imaged by SPECT reflects the static image of the cerebral blood circulation (15). Increased accumulation of IMP is generally considered to reflect hyperperfusion; this was actually reported in the affected temporal lobe with inflammation from herpes simplex virus (16). However, the present case did not show any inflammation.

According to previous reports, most brain tumors show a low uptake of IMP on ${ }^{123}$ I-IMP SPECT independent of their vascularity (9). This may be possibly because most brain tumors have few binding sites for IMP, or have no metabolic pathways which uptake and acumulate IMP. In a few excep- tional cases, brain metastasis of small cell carcinoma of the lung (17) and malignant melanoma (18), glioma (19) and meningioma (20) are reported to show high uptake of IMP only in the early images of ${ }^{123}$ I-IMP SPECT. Recently, Ohkawa et al reported a case of primary non-Hodgkin's malignant lymphoma of the brain which showed a high uptake of IMP both in the early and delayed images (21). Nakano et al also reported a dense accumulation of IMP in the delayed image in a case of primary CNS malignant lymphoma (22). In conjunction with the present case, these results suggest that the accumulation of IMP in the delayed image of ${ }^{123}$ I-IMP SPECT could be helpful in visualizing lesions of CNS malignant lymphoma.

In the present case, we finally made the diagnosis of CNS malignant lymphoma by cytologic examination of CSF. However, positive results may not always be obtained from the CSF examination (12). There have been few cases with an increased accumulation of IMP, with the exception of herpes simplex virus encephalitis and malignant lymphoma. Therefore, if inflammatory lesions such as herpes simplex virus encephalitis can be excluded, ${ }^{123}$ I-IMP SPECT is recommended as an early investigation method for patients with suspected CNS malignant lymphoma.

\section{References}

1) Neault RW, Van Scoy RE, Okazaki H, MacCarty CS. Uveitis associated with isolated reticulum cell sarcoma of the brain. Am J Opthalmol 73: 431, 1972.

2) Harstad HK, Arnesen K. Malignant lymphoma of the eye and brain. Report of a case. Acta Ophthalmol (Copenh) 52: 211, 1974

3) Parver LM, Font RL. Malignant lymphoma of the retina and brain. Arch Ophthalmol 97: 1505, 1979.

4) Rosenbaum TJ, MacCarty CS, Buettner H. Uveitis and cerebral reticulumcell sarcoma (large-cell lymphoma). J Neurosurg 50: 660, 1979.

5) Kattah JC, Jenkins RB, Pilkerton R, Rao NA, Manz HJ, Frazer M. Multifocal primary ocular and central nervous system malignant lymphoma. Ann Ophthalmol 13: 589, 1981.

6) Rockwood EJ, Zakov ZN, Bay JW. Combined malignant lymphoma of the eye and CNS (reticulum-cell sarcoma). J Neurosurg 61: 369, 1984.

7) Corriveau C, Easterbrook M, Payne D. Lymphoma simulating uveitis (masquerade syndrome). Can J Ophthalmol 21: 144, 1986.

8) Kinoshita F, Koga I, Okamura R, Noda K, Muto K. Malignant lymphoma simulating uveitis (masquerade syndrome). Nihon-Ganka-Kiyo 40: 1377, 1989 (in Japanese with English abstract).

9) LaFrance ND, Wagner HN Jr, Whitehouse P, Corley E, Duelfer T. Decreased accumulation of isopropyl-iodoamphetamine (I-123) in brain tumors. J Nucl Med 22: 1081, 1981.

10) DeAngelis LM, Yahalom J, Heinemann M-H, Cirrincione C, Thaler HT, Krol G. Primary CNS lymphoma: Combined treatment with chemotherapy and radiotherapy. Neurology 40: 80, 1990.

11) Mavligit GM, Stuckey SE, Cabanillas FF, et al. Diagnosis of leukemia or lymphoma in the central nervous system by $\beta_{2}$-microglobulin determination. N Engl J Med 303: 718, 1980.

12) Woodman R, Shin K, Pineo G. Primary non-Hodgkin's lymphoma of the brain. A review. Medicine 64: 425, 1986.

13) Enzmann DR, Krikorian J, Norman D, Kramer R, Pollock J, Faer M. Computed tomography in primary reticulum cell sarcoma of the brain. Radiology 130: 165, 1979.

14) Peretti-Viton P, Margain D, Arnaud O, Perez-Castillo AM, Graziani N. Primary and secondary lymphomas of the brain: An MRI study. J Neuroradiol 18: 173, 1991.

15) Winchell HS, Baldwin RM, Lin TH. Development of I-123-labelled 


\section{Yoshizawa et al}

amines for brain studies: Localization of I-123 iodophenylalkyl amines in rat brain. J Nucl Med 21: 940, 1980.

16) Launes J, Nikkinen $P$, Lindroth $L$, Brownell A-L, Liewendahl $K$, Iivanainen M. Diagnosis of acute herpes simplex encephalitis by brain perfusion single photon emission computed tomography. Lancet 1: 1188, 1988.

17) Szasz IJ, Lyster D, Morrison RT. Iodine-123 IMP uptake in brain metastases from lung cancer. J Nucl Med 26: 1342, 1985.

18) Naegel JS, Ichise M, Mueller MD, et al. Increased iofetamine I-123 brain uptake in metastatic melanoma. Arch Neurol 45: 1126, 1988.

19) Ell PJ, Lui D, Gullum I, Jarritt PH, Donaghy M, Harrison MJG. Cerebral blood flow studies with ${ }^{123}$ I-labelled amines. Lancet I: $1348,1983$.

20) Nishimura $T$, Hayashida $K$, Uehara $T$, et al. Two patients with meningioma visualized as high uptake by SPECT with $\mathrm{N}$-isopropyl-p-iodo-amphetamine (I-123). Neuroradiol 30: 351, 1988.

21) Ohkawa $S$, Yamadori A, Mori E, et al. A case of primary malignant lymphoma of the brain with high uptake of ${ }^{123}$ I-IMP. Neuroradiol 31: 270, 1989.

22) Nakano S, Kinoshita K, Jinnouchi S, Hoshi H, Watanabe K. Unusual uptake and retention of I-123 IMP in brain tumors. Clin Nucl Med 13: 742,1988 